COMPUTATIONAL METHODS IN APPLIED MATHEMATICS, Vol.9(2009), No. 2, pp. 113-122

(C) 2009 Institute of Mathematics of the National Academy of Sciences of Belarus

\title{
THE DETERMINANT METHOD FOR NONSELFADJOINT SINGULAR STURM — LIOUVILLE PROBLEMS
}

\author{
A. BOUMENIR ${ }^{1}$
}

\begin{abstract}
We are concerned with the computation of eigenvalues of singular nonselfadjoint Sturm — Liouville problems by the method of determinants. The representation of a differential operator by an infinite matrix allows the use of Lidskii's theorem to define its determinant. The finite section is then used to compute eigenvalues in a simple way. This direct method borrows stable methods from numerical linear algebra to compute a large number of eigenvalues with high precision. Numerical examples with nondifferentiable and complex valued coefficients are treated at the end.
\end{abstract}

2000 Mathematics Subject Classification: 34L16, 47A58.

Keywords: eigenvalues, Sturm — Liouville, Lidskii's trace theorem.

\section{Introduction}

Although the computation of eigenvalues is classical, there still remain challenging computational problems in the area of singular Sturm — Liouville (SL) operators [1]. This note is motivated by the following computational issue: how can we compute eigenvalues of singular nonselfadjoint SL operators defined by

$$
l(y):=-\left(p(x) y^{\prime}(x, \lambda)\right)^{\prime}+q(x) y(x, \lambda)=\lambda w(x) y(x, \lambda), \quad-\infty \leqslant a<x<b \leqslant \infty,
$$

where the coefficient $q(x) \in \mathbb{C}$ is complex valued, $1 / p, q, w \in L^{l o c}(a, b)$ and $p, w>0$ with minimal numerical integration. For regular problems [4], interpolation is used to reconstruct the characteristic function, whose zeros are the eigenvalues, from a few of its values by sampling. Because the problem is regular, we could use numerical integration to generate the a few samples. However, when the coefficients in (1.1) are not smooth, then numerical integration and consequently the shooting method may not be able to provide those sample points for interpolation. A typical class of singular differential operators, for example, would be Legendre type Sturm — Liouville operators,

$$
\left\{\begin{array}{l}
L(y):=-\left(\left(1-x^{2}\right) y^{\prime}(x)\right)^{\prime}+q(x) y(x)=\lambda y(x), \quad-1<x<1 \\
\lim _{x \rightarrow \pm 1}\left(1-x^{2}\right) y^{\prime}(x)=0
\end{array}\right.
$$

where $q \in L(-1,1)$ and $q(x)$ is complex valued, say $q(x)=\ln \left(x^{2}\right)+I \sin (1 / x)$. Clearly, at the singular end $x=-1$, the derivative cannot be computed numerically and initial value problems are not easy to set. Also known methods such as the Prüffer method and shooting

\footnotetext{
${ }^{1}$ Department of Mathematics, University of West Georgia, Carrollton, GA 30118, USA. E-mail: boumenir@westga.edu
} 
methods are not applicable since the operator is not self-adjoint, which means the eigenvalues are scattered in the complex plane [3]. Thus, the need for a method that does not rely on numerical integration of initial value problems arises.

For nonselfadjoint problems, Osborn [9] first considered operators $\widetilde{L}=L+A$, where $L^{-1}$ is a positive Hilbert - Schmidt operator and $\|A\|<\lambda_{1}$, where $\lambda_{n}$ are the eigenvalues of $L$. A Gershgorin type theorem is first proven, that is, eigenvalues are in the disks $C_{m}=\{\lambda$ : $\left.\left|\lambda-\lambda_{m}\right| \leqslant\|A\|\right\}$. The proof uses the argument principle to detect the presence of eigenvalues inside $C_{m}$ by looking at the contour integral

$$
N(t)=\frac{1}{2 \pi i} \int_{\partial C_{m}} \frac{\delta_{t}^{\prime}(\lambda)}{\delta_{t}(\lambda)} d \lambda,
$$

where $\delta_{t}$ is a determinant function of the operator $L+t A$, and $0 \leqslant t \leqslant 1$. In the case of differential operators, this determinant could simply be a characteristic function, i.e., a function whose zeros are the eigenvalues, such as $\delta_{0}(\lambda)=(1 / \sqrt[4]{\lambda}) \sin (\sqrt[4]{\lambda}) \sinh (\sqrt[4]{\lambda})$ for $L(y)(x)=y^{(4)}(x), 0 \leqslant x \leqslant 1$ under the boundary condition $y(0)=y^{\prime \prime}(0)=y(1)=y^{\prime \prime}(1)=$ 0 (see example (a) in [10]). In the case of infinite matrices, then $\delta_{t}(\lambda)$ is the Fredholm determinant of the Hilbert - Schmidt operator $(L+t A)^{-1}$. By using a computable error bound on the radii of the disks, Osborn could minimize the enclosures on the eigenvalues of $\widetilde{L}$ by taking the smallest disk. In [10], the results are then extended to unbounded operators $A$, such that $\sum_{i \geqslant 1}\left|\left(A y, x_{i}\right)\right| / S_{i} \leqslant\|y\|$ holds for all $y$ in the domain of $A$ and for a certain sequence $\left(S_{i}\right)_{i \geqslant 1}$.

In this note we also look at operators $\widetilde{L}=L+A$, but $L^{-1}$ is a positive trace class and $A$ is an unbounded operator. The advantage of trace class operators is Lidskii's theorem, which defines the determinant in a very simple and direct way [8]. Recall that if $K$ is a trace class with eigenvalues $\lambda_{n}$, then $\operatorname{det}(1-K)=\prod_{n \geqslant 0}\left(1-\lambda_{n}\right)$. In the case of a Hilbert - Schmidt operator, say $H$, the determinant is defined by $\operatorname{det}_{2}(1+H)=\operatorname{det}[(1+H) \exp (-H)]$, which is more difficult to compute (see [5]). The unboundedness of the operator $A$ is described in terms of the potential $q,\left\|q(x) \varphi_{n}\right\| \lambda_{n}^{-\beta} \in \ell^{2}$ instead of $A$. The main reason is that an estimate on the matrix may not be applicable to the multiplication operator $q$.

Legendre type operators are typical singular SL for which Lidskii's determinant is applicable since their large eigenvalues are close to $n^{2}+n$. On the numerical side, the simple fact that Legendre polynomials are defined through a 3 term recursion formula leads to structured matrices, which simplifies significantly the process of filling up the matrix. For example, the entries which are integrals can be computed symbolically with no round-off error, and obviously a finite determinant makes use of algebraic operations only, and so there are no round-off errors when a computer algebra system is used

The main motivation stems from applications. In control theory, for stability, the spectrum of nonselfadjoint operators is usually required to be on the left hand side of the complex plane, i.e., $\operatorname{Re}\left(\lambda_{\mathrm{n}}\right) \leqslant 0$. In order to control a process in real time, engineers look for a reliable and fast algorithm to compute and track the evolution of these eigenvalues. In medical imaging [7], Colton and Monk show how the eigenvalues of the scattering operator, which is compact, can be used in the detection of leukemia. The diagnosis for Leukemia then reduces to whether the eigenvalues of the scattering operator are contained in a certain unit disk off the real line. Unfortunately, no numerical code was accurate enough for the numerical experiments to be conclusive. We believe that the error estimates from Lidskii's theorem [8] would help obtain guaranteed bounds for eigenvalues. Recall that most numerical integrators would require smooth coefficients, which we do not assume. Our algorithm is designed 
to handle integrable but nowhere differentiable coefficients such as $q(x)=\sum_{n \geqslant 1} n^{-0.52} e^{i 2 n \pi x}$ or $q(x)=\sum_{n \geqslant 1} n^{-0.52} \mathbb{P}_{n}(x)$, where $\mathbb{P}_{n}$ are the normalized Legendre polynomials. Clearly, in both cases $q \in L^{2}(-1,1)$ and is nowhere differentiable, and when the Fourier coefficients of $q$ are available, then the entries of the matrix associated with $q$ can be computed without numerical integration.

\section{Preliminaries}

Consider the singular SL operator defined by

$$
L y(x)=\frac{-1}{w(x)}\left(\left(p(x) y^{\prime}(x)\right)^{\prime}+q(x) y(x)\right),
$$

where $a<x<b$. As usual for the right definite case, we assume $\frac{1}{p}, q, w \in L^{l o c}(a, b)$ and $p, w>0$. Denote the maximal domain for the operator $L$ by

$$
D_{\max }=\left\{f \in L_{w}^{2}(a, b): p f^{\prime} \in A C^{l o c}(a, b) \text { and } L f \in L_{w}^{2}(a, b)\right\},
$$

where $A C^{l o c}(a, b)$ is the space of absolutely continuous functions over $[c, d] \subset(a, b)$ and

$$
L_{w}^{2}(a, b)=\left\{f \text { measurable: } \int_{a}^{b}|f(x)|^{2} w(x) d x<\infty\right\} .
$$

The domain of any operator generated by $L$ and acting in $L_{w}^{2}(a, b)$ is a restriction of $D_{\max }$, which could be obtained by using boundary functionals. For example, if $L$ is in the limiting circle case, then we need two boundary conditions

$$
D_{L}=\left\{f \in D_{\max }: B_{a}(f)=0, \quad B_{b}(f)=0\right\} \subset D_{\max }
$$

and the spectrum is discrete. In the case $L \neq L^{*}$, i.e., $L$ is a nonselfadjoint operator, then by an eigenvalue is meant a value $\lambda$ for which $L y=\lambda y$ has a nontrivial solution which belongs to $D_{L}$. In all that follows we assume that the spectrum of $L$ is purely discrete, i.e., contains eigenvalues only.

Since we are working in the separable Hilbert space $H:=L_{w}^{2}(a, b)$, let us denote by $\left\{\varphi_{n}\right\}_{n \geqslant 0}$ one of its orthonormal bases, i.e., for any $f \in H$ then $f=\sum_{n \geqslant 0} c_{n} \varphi_{n}$, where $\left\|\varphi_{n}\right\|=1$ and in order to handle solutions in the domain $D_{L}$, we assume that $\left\{\varphi_{n}\right\}_{n \geqslant 0} \subset$ $D_{L}$. Denote the inner products

$$
(f, g)_{w}=\int_{a}^{b} f(x) \overline{g(x)} w(x) d x, \quad(f, g)=\int_{a}^{b} f(x) \overline{g(x)} d x .
$$

The operator $L$ acting in $L_{w}^{2}(a, b)$ can then be represented by an infinite matrix $\mathbb{A}$, whose entries are

$$
a_{n k}=\left(L \varphi_{k}, \varphi_{n}\right)_{w}=-\left(\left(p \varphi_{k}^{\prime}\right)^{\prime}, \varphi_{n}\right)+\left(q \varphi_{k}, \varphi_{n}\right), \quad \mathbb{A}=\mathbb{M}+\mathbb{Q},
$$

where $\mathbb{M}$ and $\mathbb{Q}$ are infinite matrices. To proceed further, denote by $T$ the isometry $H \rightarrow \ell^{2}$ defined by

$$
T(f)=\left(c_{n}\right)_{n \geqslant 0},
$$


where $c_{n}=\left(f, \varphi_{n}\right)_{w}$ and $\ell^{2}:=\left\{\left(c_{n}\right)_{n \geqslant 0}: \sum_{n \geqslant 0}\left|c_{n}\right|^{2}<\infty\right\}$. The Parseval equality yields $\|T f\|^{2}=\sum_{n \geqslant 0}\left|c_{n}\right|^{2}=\|f\|^{2}$. Since the operators $\mathbb{A}$ and $L$ are isometric, they both have the same spectrum, and obviously the spectrum of $\mathbb{A}$ should be easier to compute. Denote by $\mathbb{S}_{n}$ the set of compact operators acting in $\ell^{2}$ such that

$$
\mathbb{S}_{n}:=\left\{\mathbf{K} \text { compact }:\|\mathbf{K}\|_{n}:=\left(\sum_{j \geqslant 0} s_{j}^{n}(\mathbf{K})\right)^{1 / n}<\infty\right\},
$$

where $s_{j}(\mathbf{K})$ are the singular values of $\mathbf{K}$. The class $\mathbb{S}_{1}$ is called the trace class while $\mathbb{S}_{2}$ is the Hilbert - Schmidt class of compact operators. Also denote by $P_{n}$ the finite rank projection operator in $\ell^{2}$

$$
P_{n}\left(\left(c_{k}\right)_{k \geqslant 0}\right)=\left(c_{0}, c_{1}, c_{2}, \ldots, c_{n}, 0,0, \ldots\right) .
$$

Without loss of generality, we can assume that the matrix $\mathbb{M}$ has an inverse, otherwise translate its spectrum to avoid the value zero. A further assumption which would simplify the proof is that $\mathbb{M}$ is self-adjoint and positive, so its fractional powers $\mathbb{M}^{-\alpha}$ are well defined.

Proposition 2.1. Assume that $\mathbb{M}^{-1}, \mathbb{M}^{-\alpha} \mathbb{Q M}^{\alpha-1} \in \mathbb{S}_{1}$, where $\alpha>0$, then

$$
\lambda \in \sigma_{\mathbb{A}} \Longleftrightarrow \lim _{n \rightarrow \infty} \operatorname{det}\left(1+P_{n} \mathbb{M}^{-1 / 2} \mathbb{Q M}^{-1 / 2} P_{n}-\lambda P_{n} \mathbb{M}^{-1} P_{n}\right)=0
$$

Proof. If $\lambda$ is an eigenvalue, $\lambda \in \sigma_{\mathbb{A}}$, then there exists $c \in \ell^{2}, c \neq 0$ such that

$$
\mathbb{M} c+\mathbb{Q} c=\lambda c .
$$

Premultiplying by $\mathbb{M}^{-\alpha}$ and set $c=\mathbb{M}^{\alpha-1} \widetilde{c}$ to obtain

$$
\widetilde{c}+\mathbb{M}^{-\alpha} \mathbb{Q M}^{\alpha-1} \widetilde{c}=\lambda \mathbb{M}^{-1} \widetilde{c}
$$

The above identity now reads as

$$
\left(1+\mathbb{M}^{-\alpha} \mathbb{Q M}^{\alpha-1}-\lambda \mathbb{M}^{-1}\right) \widetilde{c}=0
$$

where $\mathbb{M}^{-\alpha} \mathbb{Q} \mathbb{M}^{\alpha-1}-\lambda \mathbb{M}^{-1} \in \mathbb{S}_{1}$ and thus by $[8$, Theorem II.6.1] we have

$$
\Delta(\lambda):=\operatorname{det}\left(1+\mathbb{M}^{-1 / 2} \mathbb{Q} \mathbb{M}^{-1 / 2}-\lambda \mathbb{M}^{-1}\right)=0
$$

and furthermore the eigenvalues of $\mathbb{A}$ and the zeros of $\Delta$ coincide. Then use a variant of $[8$, Theorem IV.5.2] to see that $\Delta$ is in fact an entire function of $\lambda$. We now show that $\Delta$ in (2.1) can be approximated by determinants of finite sections. To this end, recall that the mapping $K \rightarrow \operatorname{det}(1+K)$ is a continuous function over $\mathbb{S}_{1}$, [8, Theorem IV.5.2],

$$
|\operatorname{det}(1+C)-\operatorname{det}(1+E)| \leqslant\|C-E\|_{1} \exp \left(1+\|C\|_{1}+\|E\|_{1}\right)
$$

where $C$ and $E$ are compact operators in $\mathbb{S}_{1}$. Also from the pointwise convergence, $P_{n} x \rightarrow x$ for any $x \in \ell^{2}$, and for any fixed $\lambda$ in the complex plane we have [8, Theorem 5.5, p. 63]

$$
P_{n} \mathbb{M}^{-\alpha} \mathbb{Q M}^{\alpha-1} P_{n}-\lambda P_{n} \mathbb{M}^{-1} P_{n} \stackrel{\mathbb{S}_{1}}{\longrightarrow} \mathbb{M}^{-\alpha} \mathbb{Q M}^{\alpha-1}-\lambda \mathbb{M}^{-1} \text { as } n \rightarrow \infty
$$

in other words,

$$
\left(1-P_{n}\right) \mathbb{M}^{-\alpha} \mathbb{Q M}^{\alpha-1}\left(1-P_{n}\right) \stackrel{\mathbb{S}_{1}}{\longrightarrow} 0 \text { and }\left(1-P_{n}\right) \mathbb{M}^{-1}\left(1-P_{n}\right) \stackrel{\mathbb{S}_{1}}{\longrightarrow} 0 \quad \text { as } n \rightarrow \infty .
$$


If we denote the polynomials

$$
\Delta_{n}(\lambda):=\operatorname{det}\left(1+P_{n} \mathbb{M}^{-\alpha} \mathbb{Q M}^{\alpha-1} P_{n}-\lambda P_{n} \mathbb{M}^{-1} P_{n}\right),
$$

then by (2.2) and (2.3) we have

$$
\Delta_{n}(\lambda) \rightarrow \Delta(\lambda) \text { as } n \rightarrow \infty
$$

and

$$
\left|\Delta_{n}(\lambda)-\Delta(\lambda)\right| \leqslant m(\lambda)\left\|\left(1-P_{n}\right)\left(\mathbb{M}^{-\alpha} \mathbb{Q M}^{\alpha-1}-\lambda \mathbb{M}^{-1}\right)\left(1-P_{n}\right)\right\|_{1}
$$

where

$$
m(\lambda):=\exp \left(1+\left\|P_{n} \mathbb{M}^{-\alpha} \mathbb{Q M}^{\alpha-1} P_{n}-\lambda P_{n} \mathbb{M}^{-1} P_{n}\right\|_{1}+\left\|\mathbb{M}^{-\alpha} \mathbb{Q M}^{\alpha-1}-\lambda \mathbb{M}^{-1}\right\|_{1}\right)
$$

From the fact that

$$
\left\|P_{n} \mathbb{M}^{-\alpha} \mathbb{Q M}^{\alpha-1} P_{n}-\lambda P_{n} \mathbb{M}^{-1} P_{n}\right\|_{1} \leqslant\left\|\mathbb{M}^{-\alpha} \mathbb{Q M}^{\alpha-1}-\lambda \mathbb{M}^{-1}\right\|_{1}
$$

we have

$$
m(\lambda) \leqslant \exp \left(1+2\left\|\mathbb{M}^{-\alpha} \mathbb{Q M}^{\alpha-1}-\lambda \mathbb{M}^{-1}\right\|_{1}\right)
$$

Next, if we now restrict $\lambda$ to any compact domain of the complex plane, $\Omega$ say, then (2.6) leads to

$$
\sup _{\lambda \in \Omega} m(\lambda) \leqslant \exp \left(1+2\left\|\mathbb{M}^{-\alpha} \mathbb{Q M}^{\alpha-1}\right\|_{1}+w\left\|\mathbb{M}^{-1}\right\|_{1}\right)
$$

where $w=\sup _{\lambda \in \Omega}|\lambda|$ and

$$
\begin{gathered}
\sup _{\lambda \in \Omega}\left|\Delta_{n}(\lambda)-\Delta(\lambda)\right| \leqslant \sup _{\lambda \in \Omega} m(\lambda) \sup _{\lambda \in \Omega}\left\|\left(1-P_{n}\right)\left(\mathbb{M}^{-\alpha} \mathbb{Q M}^{\alpha-1}-\lambda \mathbb{M}^{-1}\right)\left(1-P_{n}\right)\right\|_{1} \leqslant \\
\sup _{\lambda \in \Omega} m(\lambda)\left\{\left\|\left(1-P_{n}\right)\left(\mathbb{M}^{-\alpha} \mathbb{Q M}^{\alpha-1}\right)\left(1-P_{n}\right)\right\|_{1}+w\left\|\left(1-P_{n}\right) \mathbb{M}^{-1}\left(1-P_{n}\right)\right\|_{1}\right\} .
\end{gathered}
$$

Thus we deduce from (2.4) that $\sup _{\lambda \in \Omega}\left|\Delta_{n}(\lambda)-\Delta(\lambda)\right| \rightarrow 0$ as $n \rightarrow \infty$. Each zero of $\Delta$, say of multiplicity $k$, can be enclosed in a small enough disk, say $D$. Now by Hurwitz's theorem, there is an $N(D)$ such that for each $n \geqslant N(D) \Delta_{n}$ has the same number of zeros as $\Delta$ in $D$, i.e., $k$ zeros. Therefore, any eigenvalue of $\mathbb{A}$, which is a zero of $\Delta$ in $\mathbb{C}$, can be approximated by the zeros of $\Delta_{n}$.

Observe that when $\mathbb{M}$ is diagonal, then the determinant of these finite matrices is easier to compute. Assume that $\mathbb{M}=\operatorname{diag}\left(\mu_{\mathrm{n}}\right)_{\mathrm{n} \geqslant 0}$ and $\mu_{n}>0$, in other words, the basis $\left\{\varphi_{n}\right\}_{n \geqslant 0}$ is made of the eigenfunctions of the operator $\mathbb{M}$, then $\mathbb{M}^{-1}=\operatorname{diag}\left(\mu_{\mathrm{n}}^{-1}\right)_{\mathrm{n} \geqslant 0}$ and $P_{n} \mathbb{M}^{-1}=$ $\mathbb{M}^{-1} P_{n}$. Thus, we can factor the finite determinant

$$
\begin{gathered}
\Delta_{n}(\lambda)=\operatorname{det}\left(1+P_{n} \mathbb{M}^{-\alpha} \mathbb{Q M}^{\alpha-1} P_{n}-\lambda P_{n} \mathbb{M}^{-1} P_{n}\right)= \\
\operatorname{det}\left(P_{n} \mathbb{M}^{-\alpha} P_{n}\right) \operatorname{det}\left(P_{n} \mathbb{M} P_{n}+P_{n} \mathbb{Q} P_{n}-\lambda I_{n}\right) \operatorname{det}\left(P_{n} \mathbb{M}^{\alpha-1} P_{n}\right)= \\
\left(\prod_{k=0}^{n} \frac{1}{\mu_{k}}\right) \operatorname{det}\left(P_{n} \mathbb{M} P_{n}+P_{n} \mathbb{Q} P_{n}-\lambda I_{n}\right)=\prod_{k=0}^{n} \frac{1}{\mu_{k}} \operatorname{det}\left(P_{n}(\mathbb{M}+\mathbb{Q}) P_{n}-\lambda I_{n}\right) .
\end{gathered}
$$

It is readily seen that although the functions $\Delta_{n}$ and $\operatorname{det}\left(P_{n}(\mathbb{M}+\mathbb{Q}) P_{n}-\lambda I_{n}\right)$ are different, their zeros coincide. These are nothing but the eigenvalues of the finite section of the matrix $\mathbb{M}+\mathbb{Q}$ and this establishes a direct connection between the Rayleigh — Ritz method and the determinant. Since the inverses of $\mathbb{M}$ and $\mathbb{Q}$ are not needed, this yields a low cost computation. We now prove 
Proposition 2.2. Assume that $\mathbb{M}$ is a diagonal matrix with eigenvalues $\mu_{n}>0$ such that $\sum_{n \geqslant 0} \mu_{n}^{-1}<\infty$ and $\mathbb{Q}$ is a bounded operator acting in $\ell^{2}$. Then $\forall \lambda_{k} \in \sigma_{L}, \exists \lambda_{k}(n)$ such that $\operatorname{det}\left(P_{n}(\mathbb{M}+\mathbb{Q}) P_{n}-\lambda_{k}(n) I_{d}\right)=0$ and $\lambda_{k}(n) \rightarrow \lambda_{k}$ as $n \rightarrow \infty$.

Proof. It is enough to see that $\mathbb{M}^{-1} \in \mathbb{S}_{1}$ while $\mathbb{M}^{-1 / 2} \in \mathbb{S}_{2}$. Thus, $\mathbb{Q} \mathbb{M}^{-1 / 2} \in \mathbb{S}_{2}$ and the product $\mathbb{M}^{-1 / 2} \mathbb{Q M}^{-1 / 2} \in \mathbb{S}_{1}$, as the product of two Hilbert — Schmidt operators is a trace class. Thus, the conditions of Proposition 2.1 hold.

We next recall that the condition for $\mathbb{Q}$ is bounded.

Proposition 2.3. Let $q$ be the multiplication operator $L_{w}^{2}(a, b) \rightarrow L_{w}^{2}(a, b)$ with $q \in$ $L^{\infty}(a, b)$. Then $\mathbb{Q}$ is a bounded operator in $\ell^{2}$.

Proof. It is enough to see that $f \rightarrow q f$ is a bounded operator in $L_{w}^{2}(a, b)$

$$
\int_{a}^{b}|q(x) f(x)|^{2} w(x) d x \leqslant \operatorname{esssup}_{a \leqslant x \leqslant b}|q(x)|^{2} \int_{a}^{b}|f(x)|^{2} w(x) d x .
$$

Since the matrix $\mathbb{Q}$ is isometric to the multiplication by $q$, it follows that $\mathbb{Q}$ is also a bounded operator in $\ell^{2}$.

In differential equations, we usually need $q \in L^{1}(a, b)$ or $q \in L^{2}(a, b)$, which means that $q$ may not be a bounded operator.

Proposition 2.4. Assume that $\mathbb{M}=\operatorname{diag}\left(\mu_{\mathrm{n}}\right)_{\mathrm{n} \geqslant 0}$ where $0<\mu_{n} \uparrow \infty$ and for a certain $1>2 \alpha \geqslant \frac{1}{2}$, we have $\mu_{n}^{2 \alpha-1} \in \ell^{2}$, together with $\left\|q(x) \varphi_{n}(x)\right\| \mu_{n}^{-2 \alpha} \in \ell^{2}$. Then both $\mathbb{M}^{-\alpha} \mathbb{Q M}^{\alpha-1} \in \mathbb{S}_{1}$, and $\mathbb{M}^{-1} \in \mathbb{S}_{1}$. Thus, the eigenvalues of $L$ can be approximated by the eigenvalues of $P_{n}(\mathbb{M}+\mathbb{Q}) P_{n}$.

Proof. To prove that $\mathbb{M}^{-\alpha} \mathbb{Q} \mathbb{M}^{-\alpha} \mathbb{M}^{2 \alpha-1}=\mathbb{M}^{-\alpha} \mathbb{Q M}^{\alpha-1} \in \mathbb{S}_{1}$, we only need to show that both $\mathbb{M}^{-\alpha} \mathbb{Q M}^{-\alpha} \in \mathbb{S}_{2}$ and $\mathbb{M}^{2 \alpha-1} \in \mathbb{S}_{2}$ are Hilbert — Schmidt operators. Observe that $\mathbb{M}^{2 \alpha-1}=\operatorname{diag}\left(\mu_{n}^{2 \alpha-1}\right)_{n \geqslant 0}$ exists and is a Hilbert - Schmidt operator, since $\mu_{n}^{2 \alpha-1} \in \ell^{2}$. To see that $\mathbb{M}^{-\alpha} \mathbb{Q M}^{-\alpha} \in \mathbb{S}_{2}$, let us denote its entries by

$$
\widetilde{Q}_{i j}=\mu_{i}^{-\alpha} \int_{a}^{b} q(x) \varphi_{i}(x) \varphi_{j}(x) w(x) d x \mu_{j}^{-\alpha} .
$$

Clearly, we have

$$
\begin{gathered}
\sum_{i, j \geqslant 0}\left|\widetilde{Q}_{i j}\right|^{2} \leqslant \sum_{i, j \geqslant 0} \mu_{i}^{-2 \alpha}\left|\int_{a}^{b} q(x) \varphi_{i}(x) \varphi_{j}(x) w(x) d x\right|^{2} \mu_{j}^{-2 \alpha} \leqslant \\
\sum_{i, j \geqslant 0} \mu_{i}^{-4 \alpha}\left|\int_{a}^{b} q(x) \varphi_{i}(x) \varphi_{j}(x) w(x) d x\right|^{2} \sum_{i, j \geqslant 0} \mu_{j}^{-4 \alpha}\left|\int_{a}^{b} q(x) \varphi_{i}(x) \varphi_{j}(x) w(x) d x\right|^{2} .
\end{gathered}
$$

Observe that

$$
\begin{gathered}
\sum_{i, j \geqslant 0} \mu_{i}^{-4 \alpha}\left|\int_{a}^{b} q(x) \varphi_{i}(x) \varphi_{j}(x) w(x) d x\right|^{2}=\sum_{i \geqslant 0} \mu_{i}^{-4 \alpha} \sum_{j \geqslant 0}\left|\int_{a}^{b} q(x) \varphi_{i}(x) \varphi_{j}(x) w(x) d x\right|^{2}= \\
\sum_{i \geqslant 0} \mu_{i}^{-4 \alpha}\left\|q(x) \varphi_{i}(x)\right\|^{2}<\infty .
\end{gathered}
$$


Thus, $\sum_{i, j \geqslant 0}\left|\widetilde{Q}_{i j}\right|^{2}<\infty$ and so $\mathbb{M}^{-\alpha} \mathbb{Q M}^{-\alpha} \in \mathbb{S}_{2}$, i.e., Hilbert — Schmidt operators. Then $\mathbb{M}^{-\alpha} \mathbb{Q M}^{-\alpha} \times \mathbb{M}^{2 \alpha-1} \in \mathbb{S}_{1}$ since the product of two Hilbert - Schmidt operators is a trace class, we have

$$
\mathbb{M}^{-\alpha} \mathbb{Q} \mathbb{M}^{\alpha-1} \in \mathbb{S}_{1}
$$

Next to see that $\mathbb{M}^{-1} \in \mathbb{S}_{1}$, i.e. $\sum_{j \geqslant 0} \mu_{j}^{-1}<\infty$, we use $4 \alpha \geqslant 1$ to deduce that

$$
0<\mu_{j}^{-1}=\mu_{j}^{-4 \alpha+1} \mu_{j}^{4 \alpha-2} \leqslant c \mu_{j}^{4 \alpha-2}
$$

and $\mathbb{M}^{-1} \in \mathbb{S}_{1}$. Therefore $\lambda$ is an eigenvalue of $L$ if and only if

$$
\operatorname{det}\left(1+\mathbb{M}^{-1 / 2} \mathbb{Q M}^{-1 / 2}-\lambda \mathbb{M}^{-1}\right)=0
$$

which can be uniformly approximated by $\operatorname{det}\left(1+P_{n} \mathbb{M}^{-1 / 2} \mathbb{Q} \mathbb{M}^{-1 / 2} P_{n}-\lambda P_{n} \mathbb{M}^{-1} P_{n}\right)$ (see proof of Proposition 2.1).

In the next section, as an example, we study one family of not self-adjoint singular Sturm — Liouville problems generated by the Legendre differential operator.

\section{The Legendre polynomials}

Consider the Legendre differential expression

$$
-\left(\left(1-x^{2}\right) y^{\prime}(x)\right)^{\prime}=\lambda y(x)-1<x<1,
$$

whose known solutions, for non-real $\lambda$, are explicitly given by $c_{1} P_{\mu}(x)+c_{2} P_{\mu}(-x)$, where $c_{i}$, for $i=1,2$ are constants and $P_{\mu}$ is the hypergeometric series

$$
P_{\mu}(x)=F\left(\mu+1,-\mu, 1 ; \frac{1-x}{2}\right),
$$

where $\lambda=\mu(\mu+1)$. The boundary conditions are derived from the fact that as $x \rightarrow-1^{+}$ (see $[2])$,

$$
P_{\mu}(x) / \ln \left(\frac{1-x}{1+x}\right) \rightarrow-\sin (\pi \mu) / \pi \text { and }\left(1-x^{2}\right) P_{\mu}^{\prime}(x) \rightarrow 2 \sin (\pi \mu) / \pi .
$$

Since $\ln (1 \pm x) \in L^{2}(-1,1)$, it follows that the deficiency indices of the minimal operator generated by $(3.1)$ are $(2,2)$. For simplicity we add $1 / 4$ to avoid the zero eigenvalue and use the boundary conditions $\left(1-x^{2}\right) P_{\mu}^{\prime}(x) \rightarrow 0$ as $x \rightarrow \pm 1$

$$
\left\{\begin{array}{l}
L_{0}(y)(x):=-\left(\left(1-x^{2}\right) y^{\prime}(x)\right)^{\prime}+(1 / 4) y(x)=\lambda y(x), \quad-1 \leqslant x \leqslant 1 \\
\left(1-x^{2}\right) y^{\prime}(x) \rightarrow 0 \text { as } x \rightarrow \pm 1
\end{array}\right.
$$

Thus, $L_{0}$ is a self-adjoint operator in the Hilbert space $L^{2}(-1,1)$ whose eigenvalues are positive $\lambda_{n}=n^{2}+n+1 / 4=(n+1 / 2)^{2}$, where $n=0,1,2, \ldots$ The corresponding eigenfunctions are the well-known normalized Legendre polynomials of the first kind

$$
\mathbb{P}_{n}(x)=\sqrt{n+1 / 2} F\left(n+1, n-1,1 ; \frac{1-x}{2}\right)=\frac{\sqrt{n+1 / 2}}{2^{n} n !} \frac{d^{n}}{d x^{n}}\left[\left(x^{2}-1\right)^{n}\right]
$$


since series (3.2) terminates. The set $\left\{\mathbb{P}_{n}\right\}_{n \geqslant 0}$ is orthonormal in $L^{2}(-1,1)$.

Consider the eigenvalue problem

$$
\left\{\begin{array}{l}
L(y)(x):=-\left(\left(1-x^{2}\right) y^{\prime}(x)\right)^{\prime}+(1 / 4) y(x)+q(x) y(x)=\lambda y(x), \quad-1 \leqslant x \leqslant 1 \\
\left(1-x^{2}\right) y^{\prime}(x) \rightarrow 0 \text { as } x \rightarrow \pm 1
\end{array}\right.
$$

where $q \in L^{2}(-1,1)$. The matrix representation of $L$ as an operator acting in $\ell^{2}$, is given by

$$
\mathbb{A}=\mathbb{M}+\mathbb{Q},
$$

where $\mathbb{M}:=\operatorname{diag}\left(\left(\mathrm{n}+\frac{1}{2}\right)^{2}\right)_{\mathrm{n} \geqslant 0}$ and

$$
\mathbb{Q}=\left(q(x) \mathbb{P}_{k}(x), \mathbb{P}_{n}(x)\right)_{n, k \geqslant 0}
$$

is the matrix associated with $q$. In order to use Proposition 4 we need the following Lemma.

Lemma 3.1. If $q^{2}(x) /\left(1-x^{2}\right) \in L(-1,1)$, then the entries $\mathbb{Q}_{i j}=\int_{-1}^{1} q^{2}(x) \mathbb{P}_{j}(x) \mathbb{P}_{i}(x) d x$ are bounded for $i, j=0,1,2, \ldots$

Proof. The Cauchy - Schwartz inequality shows that it is sufficient to consider the case $i=j \geqslant 1$. From the inequality

$$
\left|P_{j}(x)\right| \leqslant \sqrt{\frac{\pi}{2 j\left(1-x^{2}\right)}}
$$

we have

$$
\mathbb{P}_{j}(x) \leqslant \sqrt{\frac{2 j+1}{4 j} \frac{\pi}{\left(1-x^{2}\right)}}
$$

which leads to

$$
\left\|q \mathbb{P}_{j}\right\|^{2}=\int_{-1}^{1}|q(x)|^{2}\left|\mathbb{P}_{j}(x)\right|^{2} d x \leqslant \frac{\pi(2 j+1)}{4 j} \int_{-1}^{1} \frac{|q(x)|^{2}}{\left(1-x^{2}\right)} d x .
$$

Proposition 3.1. Assume that either $q^{2}(x) /\left(1-x^{2}\right) \in L(-1,1)$ or $q \in L^{\infty}(-1,1)$. Then the eigenvalues of $L$ can be approximated by the eigenvalues of the finite matrix $P_{n}(\mathbb{M}+\mathbb{Q}) P_{n}$ as $n \rightarrow \infty$.

Proof. We only need to show that the conditions of Proposition 2 or 4 hold. We already have that $\mathbb{M}$ is positive, self-adjoint, and its eigenvalues satisfy $\sum_{n \geqslant 0}(n+1 / 2)^{-2}<\infty$, and so $\mathbb{M}^{-1} \in \mathbb{S}_{1}$. We now verify the conditions of proposition 2.4. From $\mu_{n}=(n+1 / 2)^{2}$ we obtain

$$
\mu_{n}^{2 \alpha-1}=(n+1 / 2)^{2(2 \alpha-1)} \in \ell^{2} \Rightarrow 8 \alpha<3
$$

Since $q^{2}(x) /\left(1-x^{2}\right) \in L(-1,1)$, then $\sup _{n \geqslant 0}\left\|q \mathbb{P}_{n}\right\|<\infty$, and $\left\|q \mathbb{P}_{n}\right\| \mu_{n}^{-2 \alpha} \in \ell^{2}$ implies

$$
(n+1 / 2)^{2(-2 \alpha)} \in \ell^{2} \Rightarrow 1<8 \alpha .
$$

Thus it follows that there are $\alpha$ that satisfy both $1 / 4<2 \alpha<3 / 4$ and $1 / 2 \leqslant 2 \alpha<1$ in Proposition 2.4, i.e., $1 / 2 \leqslant 2 \alpha<3 / 4$.

On the other hand, if $q \in L^{\infty}(-1,1)$ then proposition 3 implies that $\mathbb{Q}$ is a bounded operator in $\ell^{2}$ and obviously then Proposition 2 holds.

Remark 3.1. The case $q^{2}(x) /\left(1-x^{2}\right) \in L(-1,1)$ includes unbounded operators. 


\section{Examples}

We consider the following singular operator defined by

$$
\left\{\begin{array}{l}
L y:=-\left(\left(1-x^{2}\right) y^{\prime}(x)\right)^{\prime}+(1 / 4) y(x)+q(x) y(x)=\lambda y(x), \quad-1 \leqslant x \leqslant 1 \\
\lim _{x \rightarrow \pm 1}\left(1-x^{2}\right) y^{\prime}(x)=0 .
\end{array}\right.
$$

where $q(x)$ will be given in each example. We show that no numerical integration is used. The finite section generated by $\mathbb{M}$ for $n=8$, for example, is given by

$$
P_{7} \mathbb{M} P_{7}:=\frac{1}{4} \operatorname{diag}(1,9,25,49,81,121,169,225)
$$

Example 4.1. Take $q(x)=x^{7}-x^{4}$, then $P_{7} \mathbb{Q}_{7}$ is

$$
\left[\begin{array}{cccccccc}
\frac{-1}{5} & \frac{\sqrt{3}}{9} & -\frac{4 \sqrt{5}}{35} & \frac{2 \sqrt{7}}{33} & -\frac{8}{105} & \frac{8 \sqrt{11}}{429} & 0 & \frac{16 \sqrt{15}}{6435} \\
\frac{\sqrt{3}}{9} & \frac{-3}{7} & \frac{8 \sqrt{15}}{99} & -\frac{4 \sqrt{21}}{63} & \frac{16 \sqrt{3}}{143} & -\frac{8 \sqrt{33}}{693} & \frac{64 \sqrt{39}}{6435} & 0 \\
-\frac{4 \sqrt{5}}{35} & \frac{8 \sqrt{15}}{99} & \frac{-3}{7} & \frac{23 \sqrt{35}}{429} & -\frac{136 \sqrt{5}}{1155} & \frac{4 \sqrt{55}}{165} & -\frac{8 \sqrt{65}}{1001} & \frac{56 \sqrt{3}}{1683} \\
\frac{2 \sqrt{7}}{33} & -\frac{4 \sqrt{21}}{63} & \frac{23 \sqrt{35}}{429} & -\frac{13}{33} & \frac{16 \sqrt{7}}{143} & -\frac{8 \sqrt{77}}{273} & \frac{656 \sqrt{91}}{36465} & -\frac{8 \sqrt{105}}{1287} \\
-\frac{8}{105} & \frac{16 \sqrt{3}}{143} & -\frac{136 \sqrt{5}}{1155} & \frac{16 \sqrt{7}}{143} & -\frac{1929}{5005} & \frac{19 \sqrt{11}}{221} & -\frac{212 \sqrt{13}}{3003} & \frac{10066 \sqrt{15}}{230945} \\
\frac{8 \sqrt{11}}{429} & -\frac{8 \sqrt{33}}{693} & \frac{4 \sqrt{55}}{165} & -\frac{8 \sqrt{77}}{273} & \frac{19 \sqrt{11}}{221} & -\frac{521}{1365} & \frac{296 \sqrt{143}}{12597} & -\frac{196 \sqrt{165}}{9945} \\
0 & \frac{64 \sqrt{39}}{6435} & -\frac{8 \sqrt{65}}{1001} & \frac{656 \sqrt{91}}{36465} & -\frac{212 \sqrt{13}}{3003} & \frac{296 \sqrt{143}}{12597} & -\frac{71}{187} & \frac{8299 \sqrt{195}}{415701} \\
\frac{16 \sqrt{15}}{6435} & 0 & \frac{56 \sqrt{3}}{1683} & -\frac{8 \sqrt{105}}{1287} & \frac{10066 \sqrt{15}}{230945} & -\frac{196 \sqrt{165}}{9945} & \frac{8299 \sqrt{195}}{415701} & -\frac{17481}{46189}
\end{array}\right]
$$

$\mathbb{Q}$ is a band matrix since its coefficients are given by $\mathbb{Q}_{i j}=\int_{-1}^{1}\left(-x^{4}+x^{7}\right) \mathbb{P}_{j}(x) \mathbb{P}_{i}(x) d x=0$ if $8 \leqslant|j-i|$, where $i, j \geqslant 0$. Since we used the exact entries of the matrix $\mathbb{Q}$, the error is only due to the truncation of the infinite matrix. Some eigenvalues obtained from the determinant for $N=30$ and 50 are given by

\begin{tabular}{|c|c|c|c|}
\hline$N$ & $\lambda_{0}$ & $\lambda_{10}$ & $\lambda_{20}$ \\
\hline 30 & 0.0103708662351945 & 109.874079259079380 & 419.874757610736480 \\
50 & 0.0103708662351915 & 109.874079259079494 & 419.874757610734660 \\
\hline
\end{tabular}

Example 4.2. Here the real potential $q(x)=\sin (1 / x)$ is nondifferentiable and highly oscillatory at $x=0$, but is bounded. The entries of $\mathbb{Q}$ can be computed without numerical integration and are linear combinations of the numbers $\operatorname{Si}(1), \sin (1), \cos (1), \pi$ which can be approximated to any precision. For example,

$$
\int_{-1}^{1} P_{4}(x) P_{5}(x) \sin (1 / x) d x=\frac{622271}{368640} \operatorname{Si}(1)+\frac{50329}{73728} \sin (1)+\frac{391033}{368640} \cos (1)-\frac{622271}{737280} \pi
$$

and

\begin{tabular}{|c|c|c|}
\hline$N$ & $\lambda_{0}$ & $\lambda_{29}$ \\
\hline 30 & 0.0474674667282716 & 870.2552588145592 \\
60 & 0.0474499121064421 & 870.2509831859029 \\
\hline
\end{tabular}


Example 4.3. Here the potential $q(x)=(1-\mathbf{i}) *\left(1-x^{2}\right) x^{-10 / 11}$ is complex valued and satisfies $q(x) /\left(1-x^{2}\right) \in L(-1,1)$. The eigenvalues are complex and so ranking is meaningless $[2362.109510156776-16.59060143401068 * I, \quad 2172.922350508070-15.19943486722927 * I$, $1991.181625270057-14.63137874251782 * I, \quad 2452.704117132835-2.527964596485432 * I$, $1817.333454688336-14.30584516783639 * I, \quad 2258.688942177950-2.468611285330135 * I]$,

Example 4.4. Here the potential $q(x)=\mathbf{i} \ln \left(x^{2}\right)$ is also complex valued and satisfies $q^{2}(x) /\left(1-x^{2}\right) \in L(-1,1)$. Some eigenvalues are

$-.8560212272279310-.6693645194250938 * I, \quad 9.531273016378190-1.406739258667220 * I$, $16.97611211032472-1.437130849354581 * I, \quad 27.31640303824101-1.280713509081845 * I$, $39.03172259630585-1.480828981619380 * I, \quad 53.22650579024753-1.282648510409766 * I$, $87.18341985521134-1.295046074559962 * I$.

Acknowledgment. The author sincerely thanks the referee for his valuable comments.

\section{References}

1. L.D. Akulenko and S. V. Nesterov, High precision methods in eigenvalue problems and their applications, Chapman\&Hall/CRC, 2005. 1963.

2. N. Acheiser and I. Glazman, Theory of linear operators in Hilbert spaces, English Translation, Dover,

3. A. Böttcher and S. Grudsky, Toeplitz matrices, asymptotic linear algebra, and functional analysis, Birkhäuser, 2000.

4. A. Boumenir, Sampling and eigenvalues of non-self-adjoint Sturm - Liouville problems, SIAM J. Sci. Comput. 23 (2001), no. 1, pp. 219-229.

5. A. Boumenir and V.K. Tuan, The computation of eigenvalues of singular Sturm - Liouville operators, Adv. in Appl. Math. 39 (2007), no. 2, pp. 222-236.

6. F. Chatelin, The spectral approximation of linear operators with applications to the computation of eigenelements of differential and integral operators, SIAM Rev., 23 (1981), no. 4, pp. 495-522.

7. D. Colton and P. Monk, The Detection and Monitoring of Leukemia Using Electromagnetic Waves: Numerical Analysis, Inverse Problems, V.11, 1995, pp. 329-342.

8. I. Gohberg, S. Goldberg, and N. Krupnik, Traces and determinants of linear operators, Operator Theory, V.116, Birkhäuser, 2000.

9. J.E. Osborn, Approximation of the eigenvalues of non self-adjoint operators, J. Math. and Phys., 45 (1966), pp. 391-401.

10. J.E. Osborn, Approximation of the eigenvalues of a class of unbounded, nonselfadjoint operators, SIAM J. Numer. Anal., 4 (1967), pp. 45-54. 